\title{
Outpatient Intravenous Diuretic Clinic: An Effective Strategy for Management of Volume Overload and Reducing Immediate Hospital Admissions
}

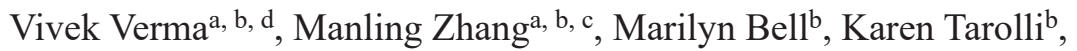 \\ Elinor Donalson ${ }^{\mathrm{b}}$, Jamie Vaughn ${ }^{\mathrm{b}}$, Gavin W. Hickey, b, c
}

\begin{abstract}
Background: Heart failure (HF) readmissions pose a major burden to patients and the healthcare system. We evaluated whether outpatient intravenous (IV) diuretic clinic is a safe and effective strategy to reduce HF hospitalizations.

Methods: We reviewed 34 clinic encounters with 27 unique patients (median age 72) who had volume overload refractory to oral diuretics that were treated with IV furosemide in the outpatient clinic. One patient $(2.9 \%)$ was admitted to the hospital directly, and the rest were discharged home.
\end{abstract}

Results: More than $80 \%$ of the patients had continued weight loss for 7 days (median weight loss $5.4 \mathrm{lb}$ ). During the median follow-up period of 15 months, 15 patients $(56 \%)$ had subsequent HF hospitalizations. HF admission was delayed by a median of 22 days from the clinic visit and 138 days from the previous HF admission prior to clinic visit. Estimated cost saving per admission avoided was $\$ 10,395$. One patient developed severe hypokalemia $(<3.0 \mathrm{mmol} / \mathrm{L})$, and the remaining had no adverse events.

Conclusion: Outpatient IV diuresis is effective and well tolerated. It leads to significant weight loss, persisting in the majority of patients for 7 days. In select patients, it should be considered as a strategy to rapidly improve symptoms, reduce hospitalizations and decrease costs.

Keywords: Heart failure; Outpatient IV diuresis; Recurrent admissions; Cost-effective; Hospitalizations

Manuscript submitted April 4, 2021, accepted April 19, 2021

Published online April 27, 2021

aDepartment of Medicine, University of Pittsburgh Medical Center, Pittsburgh, PA, USA

bVeterans Affairs Pittsburgh Healthcare System, Pittsburgh, PA, USA

${ }^{c}$ Heart and Vascular Institute, University of Pittsburgh Medical Center, Pittsburgh, PA, USA

${ }^{\mathrm{d} C}$ Corresponding Author: Vivek Verma, University of Pittsburgh Medical Center, 200 Lothrop Street, Pittsburgh, PA 15203, USA. Email: vermav@upmc.edu

doi: https://doi.org/10.14740/jocmr4499

\section{Introduction}

Heart failure (HF) is a highly prevalent medical condition in the United States, currently affecting 6.2 million patients with an estimated increase to 8.5 million by 2030 [1,2]. Despite significant advancements in pharmacological and device-based therapies in the management of HF, patients still require frequent hospitalizations for cardiovascular and non-cardiovascular conditions [3, 4]. Acute decompensated heart failure (ADHF) accounts for more than 1 million hospital admissions annually in the United States. This number has continued to rise over the last two decades [5]. Among all medical conditions, HF poses the highest risk of rehospitalization; almost one in four patients with HF requires readmission within 30 days [6]. These hospital admissions are extremely resource-intensive and over the lifetime of a patient with HF have been estimated to cost $\$ 83,900$ [7]. According to one report, almost $80 \%$ of the cost of care for HF patients can be attributed to hospital admissions [7]. Moreover, there is a direct correlation between mortality risk and hospital admission for intravenous (IV) diuresis for decompensated HF [8]. In response to increasing HF hospitalizations, the Hospital Readmission Reduction Program (HRRP) was introduced in 2012, monetarily penalizing hospitals for excessive readmissions for HF and several other medical conditions [9].

Hospitalization for HF decompensation is associated with significant increase in morbidity and mortality [8]. In order to improve patient outcomes and reduce the economic burden of management of HF on our healthcare systems, it is imperative that we focus on developing strategies to proactively prevent decompensation and manage decompensated HF in an ambulatory setting. One such initiative is administration of IV diuretics in a clinic-based setting. Although a handful of studies have been published on the use of outpatient IV diuresis for HF patients, there remains a paucity of evidence in terms of its tolerability and effectiveness in reducing hospital admissions [10-15]. In this retrospective observational study, we have evaluated the safety and efficacy of an Outpatient IV Diuretic Clinic.

\section{Materials and Methods}

\section{Clinic}

The advanced heart failure (AHF) service at the VA Pittsburgh 
Table 1. Eligibility Criteria for Outpatient IV Diuretic Clinic

\author{
Primary diagnosis of heart failure \\ Signs and symptoms of acute decompensated heart failure (weight gain, dyspnea) \\ Symptoms refractory to higher doses of oral diuretics \\ No acute cardiovascular issue (acute coronary syndrome, new arrhythmia, pulmonary embolism) \\ SBP $>100$ or at baseline SBP \\ Heart rate $50-120$ \\ Does not have NYHA class IV symptoms (dyspnea at rest, home inotropes) \\ $\mathrm{Cr} \leq 2.5$ or near baseline (increase $<0.5$ ) \\ $\mathrm{O}_{2}$ saturation $\geq 90 \%$ on $\leq 2 \mathrm{~L}$ oxygen by nasal cannula or within $2 \mathrm{~L}$ baseline oxygen requirement \\ On $\leq 260 \mathrm{mg}$ lasix daily or $10 \mathrm{mg}$ bumex daily at home \\ Labs: blood glucose $<400 \mathrm{mg} / \mathrm{dL}, \mathrm{Na}>120 \mathrm{mmol} / \mathrm{L}$, T bilirubin $<3 \mathrm{mg} / \mathrm{dL}$
}

IV: intravenous; SBP: systolic blood pressure; NYHA: New York Heart Association; Cr: creatinine.

Healthcare System (VAPHS) has a multidisciplinary team of nurses, social workers, pharmacists and physicians providing specialized care for patients diagnosed with HF. The Outpatient IV Diuretic Clinic is a division of the AHF service that was designed to provide ambulatory treatment to veterans with decompensated $\mathrm{HF}$ in efforts to help minimize the need for hospital readmission and improve their quality of life. Veterans must meet the eligibility criteria (Table 1) in order to qualify for treatment with IV diuresis in an ambulatory setting. These patients have signs and symptoms of ADHF refractory to increasing doses of oral diuretics at home. The charge nurse $(\mathrm{CN})$ at the clinic, in conjunction with the AHF team, determines if a patient is a candidate. For appropriate patients, the $\mathrm{CN}$ schedules an appointment for the same day or following day depending on clinic availability.

The clinic operates from 7 am to $7 \mathrm{pm}$. On admission to the clinic, vital signs, standing weight, electrocardiogram (EKG), basic metabolic panel (BMP), brain natriuretic peptide (BNP), digoxin level (if applicable) and magnesium level are obtained. All patients are placed on cardiac telemetry and IV access is established by the nurse. Upon return of labs treatment is initiated, typically prior to $12 \mathrm{pm}$. IV diuretics are administered as per the standardized diuretic protocol. All patients are given a cardiac diet, which limits sodium to $1,500 \mathrm{mg}$ per day and potassium to $4,700 \mathrm{mg}$ per day. They are also placed on $500 \mathrm{~mL}$ fluid restriction for $12 \mathrm{~h}$ while they are in the clinic. BMP and magnesium level are checked again at the end of the session and repeated as needed. Total urine output and weight are also recorded. At $4 \mathrm{pm}$, the AHF team assesses the patients for an appropriate disposition plan. Based on the clinical status, patients are either discharged home or admitted for observation.

The study was approved by Institution IRB. This study was conducted in compliance with the ethical standards of the responsible institution on human subjects as well as with the Helsinki Declaration.

\section{Intervention}

The clinic uses a standardized diuretic protocol designed by the AHF team based on previous studies and group consensus
[10-15]. All patients included were given IV furosemide bolus of $80-120 \mathrm{mg}$ with a 3 -h continuous infusion at the rate of 20 $\mathrm{mg} / \mathrm{h}$. The bolus dose was determined by the AHF team based on the patient's home dose of diuretics and their responses in the past during clinic visits or hospital admissions. Patients on maintenance thiazide diuretics (hydrochlorothiazide, metolazone, or chlorothiazide) were continued on their home dose in the clinic. All patients had a BMP and magnesium level checked before the initiation of diuretics and at the end of 3-h furosemide infusion. Electrolytes were repeated by the AHF team. Patients who had inadequate urine output and had stable creatinine $(\mathrm{Cr})$ after $3 \mathrm{~h}$ of IV furosemide infusion were continued at the rate of $20 \mathrm{mg} / \mathrm{h}$ for three more hours.

\section{Monitoring}

All patients were placed on cardiac telemetry to monitor for arrhythmia during the clinic visit. Heart rate (RR), blood pressure $(\mathrm{BP})$, respiratory rate $(\mathrm{RR})$ and pulse oximetry were recorded before starting diuretics, at the end of 3-h furosemide infusion, and at discharge. Standing weights were recorded at the beginning and the end of clinic stay. Urine output was collected and monitored throughout the visit. Patients who were enrolled in remote telehealth monitoring had BP, HR and standing weight recorded daily to closely monitor for further weight loss and any adverse events. Patients with cardioMEMS had pulmonary artery pressures monitored before and after IV diuresis.

\section{Outcomes}

All patients who were treated with IV diuresis at the AHF Clinic at VAPHS between January 2017 and March 2020 were included in this study. Electronic medical records were reviewed to collect data regarding patient demographics and outcomes.

The efficacy outcomes included total urine output in clinic, total weight loss (at day 0, day 2 and day 7), time to HF hospitalization or death, and time free from HF hospitalization. Weight loss on day 0 was defined as the difference be- 
tween the patient's weight at admission and at discharge from the clinic. Total weight losses on day 2 and day 7 were defined as the difference between the patient's admission weight at the clinic and the patient's weight at home on day 2 and day 7, respectively. Total time to HF hospitalization or death was determined by calculating the number of days between the clinic visit and the respective event. Time free from HF hospitalization (during follow-up period) was determined by calculating the number of days from the clinic visit to either HF hospitalization, death or end of follow-up period (March 31, 2020).

Safety outcomes included were hypotension (systolic blood pressure (SBP) $<90 \mathrm{~mm} \mathrm{Hg}$ ), electrolyte abnormalities, acute kidney injury (AKI) and same-day admission. BMP was checked towards the end of visit and hypokalemia was defined as either severe $(<3.0 \mathrm{mmol} / \mathrm{L})$ or mild-moderate $(3.0-3.5 \mathrm{mmol} / \mathrm{L})$. Increase in repeat $\mathrm{Cr}$ by $0.3 \mathrm{mg} / \mathrm{dL}$ on repeat BMP was defined as AKI. At the end of the visit, if the patient required admission for observation, it was recorded as a same-day admission.

The cost of clinic visits and the most recent HF hospitalizations prior to the clinic visit were obtained from the VAPHS data analysis center and cost accounting department. The final total cost of each clinic visit and hospital admission include cost of physician service, nursing care, other professional services, medical equipment, prescription drugs, labs, imaging, room service and other miscellaneous administrative items. The average cost per day in the hospital was calculated by dividing the total cost for hospitalization by the duration of hospital stay.

\section{Statistical analysis}

Baseline characteristics including patient demographics, comorbidities, background HF management, laboratory values and hemodynamics are presented as counts and percentages for discrete variables and median with interquartile range (IQR) for continuous data. The pre-specified efficacy and safety outcomes are summarized in tabular or graphical form using standard descriptive statistics. The Mann-Whitney U test was used to compare weight loss at day 2 and day 7 as compared to day 0 . It was also used to compare outcomes between heart failure with reduced ejection fraction (HFrEF) and heart failure with preserved ejection fraction ( $\mathrm{HFpEF}$ ) patients. A two-sided P-value $\leq 0.05$ was considered to indicate statistical significance.

\section{Results}

A total of 34 patient visits, including 27 unique patients, were included in this analysis. Four patients used the clinic more than once and two of them required weekly IV diuresis in the clinic for three consecutive weeks. The metrics related to baseline demographics, comorbidities, HF severity, pharmacological therapies and implanted devices are presented in Table 2. The median age was 72 years (IQR, 67 - 80 years) and 93\% of them were male. Fourteen patients (52\%) had HFrEF and $13(48 \%)$ had ischemic cardiomyopathy. All patients seen in the clinic had New York Heart Association (NYHA) class III symptoms. The median home diuretic dose (oral furosemide equivalent) was $200 \mathrm{mg} / 24 \mathrm{~h}$ (IQR, 160 - $260 \mathrm{mg}$ ). Twenty patients $(74 \%)$ were enrolled in remote telehealth monitoring and four $(15 \%)$ patients had CardioMEMS device in place.

The visit metrics are listed in Table 3 . The median IV furosemide dose, including bolus and continuous infusion, administered in the clinic was $180 \mathrm{mg}$ (IQR, 140 - $200 \mathrm{mg}$ ). Nineteen patients $(56 \%)$ required a single 3 -h session of furosemide infusion, and $15(44 \%)$ received an additional 3-h session. The median total urine output in the clinic was $1,500 \mathrm{~mL}$ (IQR, $1,030-2,600 \mathrm{~mL}$ ). The median weight loss at the end of the clinic visit was $2.8 \mathrm{lb}$ (IQR, 1.7 - $3.9 \mathrm{lb}$ ). The data for day 2 and day 7 weights at home were available for 19 out of the 20 patients enrolled in remote telehealth monitoring. Of those 19 patients, 18 (95\%) had further weight loss at day 2. Persistent weight loss at day 7 was seen in 17 patients $(85 \%)$. The weight loss was significantly higher on both days as compared to clinic weight loss, with median total weight loss of $5.5 \mathrm{lb}$ (IQR, 3.2 - $8.2 \mathrm{lb})$ at day $2(\mathrm{P}=0.0003)$ and $5.4 \mathrm{lb}(\mathrm{IQR}, 2.1-10.4 \mathrm{lb})$ at day $7(\mathrm{P}=0.01)$ (Fig. 1). There was no significant difference in efficacy outcomes, including urine output and weight loss, between HFrEF and HFpEF patients (Table 4).

One patient was admitted to the hospital directly from the clinic due to inadequate response to diuretics; the remainders were discharged in stable condition. Fifteen patients $(56 \%)$ have had a hospital admission for decompensated HF after the clinic visit. The median time to HF admission was 22 days (IQR, 6 - 120 days) from the clinic visit and 138 days (IQR, 41 - 328 days) from the previous $\mathrm{HF}$ admission prior to clinic visit. The remaining 12 patients (44\%) did not have any HF hospitalizations during the follow-up period. Of those 12 patients, four (15\%) died and the median time to death was 171 days (IQR, 119 - 363 days). The other eight (30\%) have had no HF admissions after the clinic visit during the median followup time of 442 days (IQR, 340 - 686 days). The median cost of a clinic visit and an HF hospitalization were \$1,076 (IQR, $\$ 960$ - \$1,233) and \$11,471 (IQR, \$4,818 - \$26,880), respectively. The median duration of hospital stay was 6 days (IQR, 3 - 8.5 days), with a median cost per day of \$1,912 (IQR, \$535 $\$ 2,987$ ) (Table 5). By preventing HF admissions in 12 patients (44\%), the estimated saving per patient was $\$ 10,395$.

There were no episodes of diuresis-related arrhythmia, AKI or hypotension during the clinic visit. Severe hypokalemia occurred in only one patient, whereas 22 patients (65\%) required potassium repletion for mild-moderate hypokalemia (Table 6).

\section{Discussion}

Despite high rates of co-morbid chronic kidney disease (CKD) and high doses of home oral diuretics, outpatient IV diuresis was safe and effective. Surprisingly, there was further persistent weight loss for up to 7 days in $85 \%$ of the patients. Twelve patients (44\%) remained alive without subsequent HF admission, and in the remainder of the patients, HF admission was delayed by a median of 22 days from the clinic visit and 138 days from the previous HF admission prior to clinic visit. This was associated with a significant cost-savings. There were no major diuresis-related metabolic or hemodynamic adverse events. 
Table 2. Patient Demographics

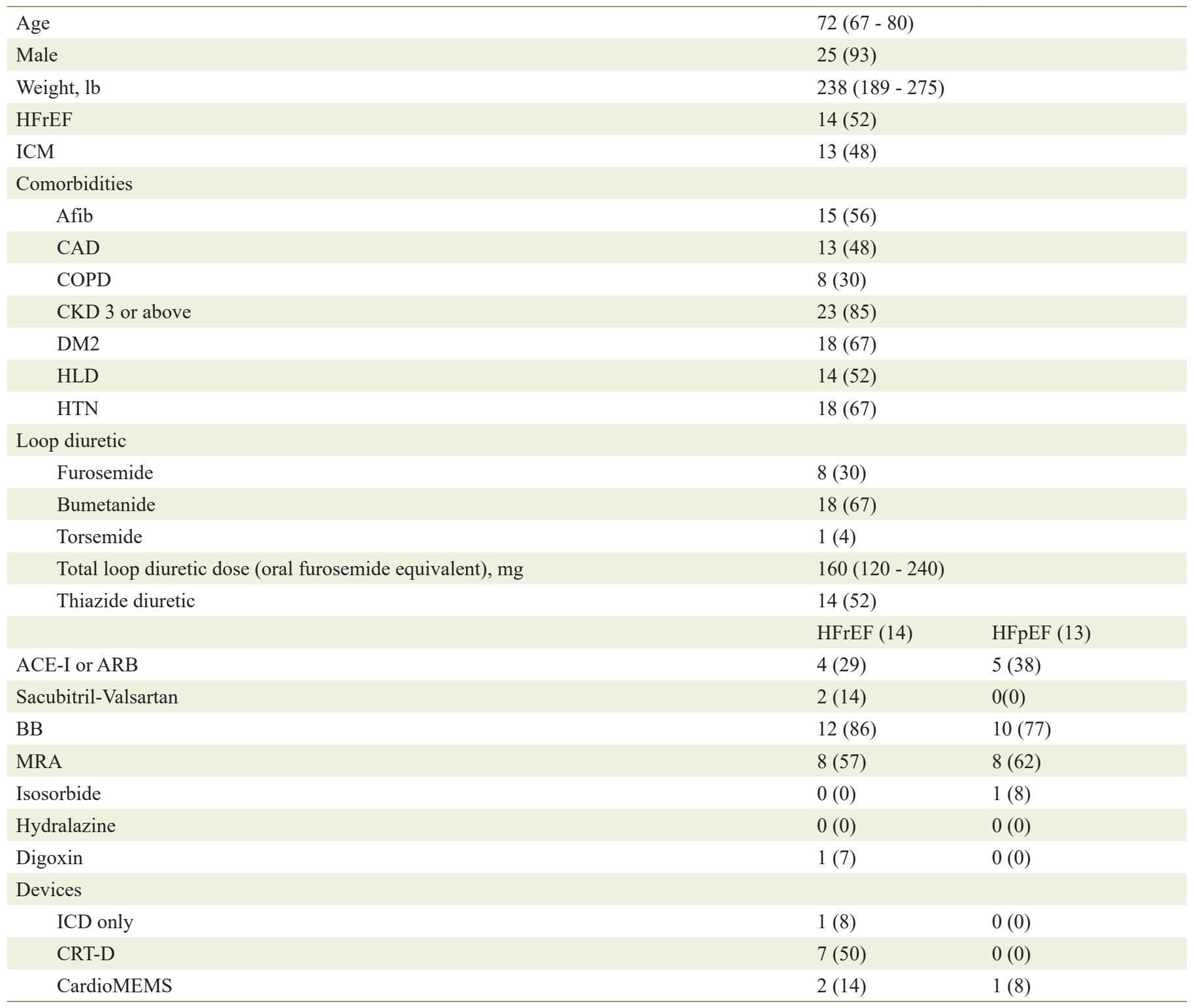

Values are represented as $\mathrm{n}(\%)$ or median (interquartile range). HFrEF: heart failure with reduced ejection fraction; ICM: ischemic cardiomyopathy; NYHA: New York Heart Association; Afib: atrial fibrillation; CAD: coronary artery disease; COPD: chronic obstructive lung disease; CKD: chronic kidney disease; DM2: diabetes mellitus type 2; HLD: hyperlipidemia; HTN: hypertension; ACE-I: angiotensin-converting enzyme inhibitor; ARB: angiotensin II receptor blocker; BB: beta-blocker; MRA: mineralocorticoid receptor antagonist; ICD: implantable cardioverter defibrillator; CRT-D: cardiac resynchronization therapy defibrillator.

While there have been several other reports on the efficacy of outpatient administration of IV diuretics, these data offer unique perspectives. Firstly, they demonstrate that there may be a role in combining multiple ambulatory strategies to reduce hospital readmissions for HF. Outpatient IV diuresis can be optimally employed in conjunction with tele-monitoring via home tele-monitoring systems or pulmonary artery (PA) pressure monitoring devices such as CardioMEMS. Although telemonitoring is a powerful tool that allows providers to intervene early if there are signs of decompensation, studies have failed to show reduction in HF admissions with this strategy alone $[16,17]$. However, when combined with IV diuresis in an outpatient clinic, tele-monitoring has the potential to significantly reduce emergency department (ED) visits and hospital admissions for HF. In this population, more than $70 \%$ of patients were enrolled in tele-monitoring. Their symptoms of HF were refractory to increasing doses of oral diuretics which would have resulted in hospitalization if not for the availability and effectiveness of Outpatient IV Diuretic Clinic. Additionally, tele-monitoring allowed for continued routine assessment after the clinic visit to evaluate for improvement in signs of HF and monitor for any potential complications. The additional 
Table 3. Visit Metrics

\begin{tabular}{|c|c|c|}
\hline & Before IV diuresis & After IV diuresis \\
\hline \multicolumn{3}{|l|}{ Lab } \\
\hline Serum sodium, $\mathrm{mmol} / \mathrm{L}$ & $138(136-139)$ & $138(136-140)$ \\
\hline Serum creatinine, mg/dL & $1.59(1.27-1.82)$ & $1.55(1.25-1.95)$ \\
\hline Serum BUN, mg/dL & $33.5(24.8-51.2)$ & $33(23-49)$ \\
\hline Systolic blood pressure, $\mathrm{mm} \mathrm{Hg}$ & $111(101.5-127.5)$ & $116(108-125)$ \\
\hline Diastolic blood pressure, $\mathrm{mm} \mathrm{Hg}$ & $67(58-75)$ & $68(62-76)$ \\
\hline Heart rate & $78(75-82)$ & $79(72-82)$ \\
\hline \multicolumn{3}{|l|}{ Clinic outcomes } \\
\hline
\end{tabular}

Values are represented as median (interquartile range). IV: intravenous; BUN: blood urea nitrogen; BNP: brain natriuretic peptide.

advantage of combining tele-monitoring and outpatient IV diuresis is that it adds to continuity of care and allows patients with advanced HF to be treated in a clinic that knows them well, rather than by new providers in the hospital that may not be familiar with the complexities of their disease. This combination of strategies, if adopted widely, has the potential to significantly improve patient outcomes as well as reduce the economic burden of HF on the healthcare system.

Many studies have failed to show significant reduction in HF hospital admissions despite close monitoring of daily weights and signs and symptoms of congestion $[17,18]$. However, novel interventions, such as guiding therapy based on changes in PA pressures using implantable hemodynamic monitoring, have been shown to successfully reduce HF hospitalizations [19]. In our analysis, only a small number of patients had CardioMEMS implanted for remote monitoring of PA pressures, making it difficult to assess the efficacy of this strategy in conjunction with outpatient IV diuresis. However, as implantable hemodynamic monitoring becomes prevalent, it will potentially enhance the efficacy of the ambulatory IV diuresis program.

The coronavirus disease 2019 (COVID-19) pandemic has accelerated the need for innovative telehealth and ambulatory

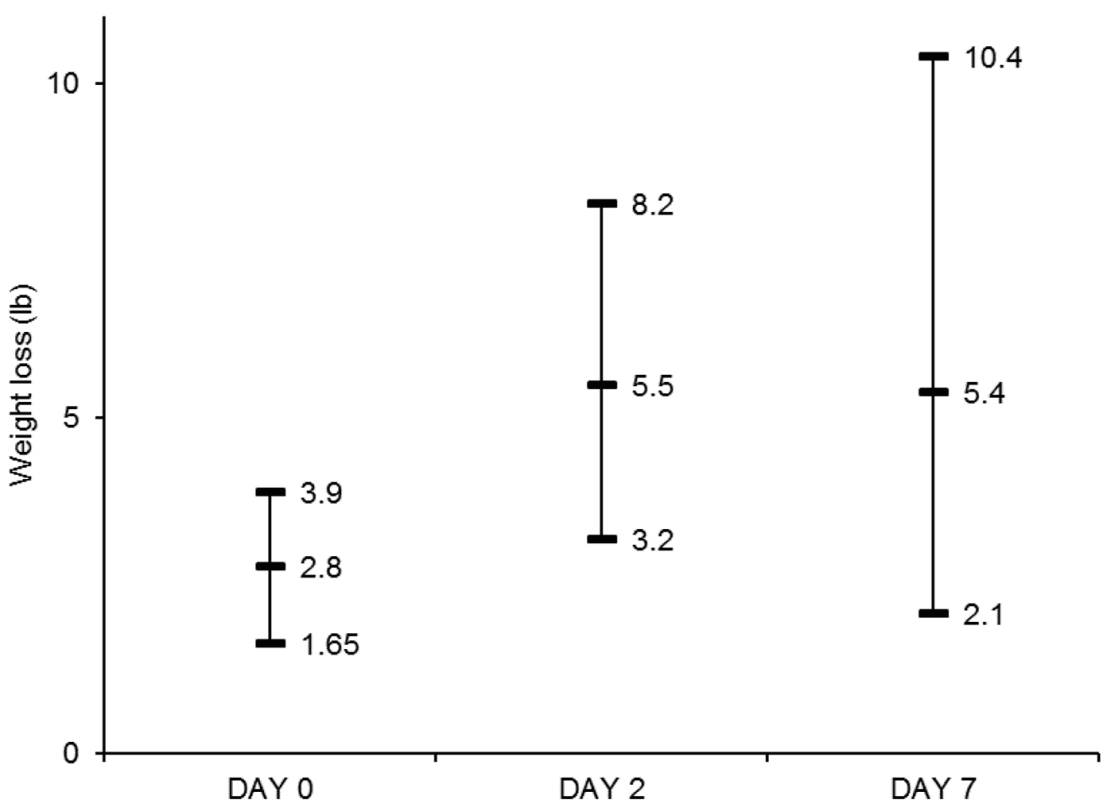

Figure 1. Median Weight loss (Ib) and interquartile range at day 0 , day 2 and day 7 . Weight losses at day 2 and day 7 were significantly higher than that at day 0 ( $\mathrm{P}<0.05$ for both comparisons). 
Table 4. Outcomes for HFrEF and HFpEF Groups

\begin{tabular}{llll}
\hline Outcomes & HFrEF* $(\mathbf{N}=\mathbf{1 4})$ & HFpEF* $(\mathbf{N}=\mathbf{1 3})$ & P value \\
\hline $\begin{array}{l}\text { Urine output, } \mathrm{mL} \\
\text { Weight loss, lb }\end{array}$ & $1,350(935-1,900)$ & $1,686(1,362-2,637)$ & 0.25 \\
$\quad$ & & $2.8(1.9-4.4)$ & 0.65 \\
$\quad$ Day 0 & $2.6(1.6-3.7)$ & $5.4(3.8-7.9)$ & 0.53 \\
$\quad$ Day 2 & $7.1(3.2-11.4)$ & $4.6(2.3-8.4)$ & 0.51 \\
\hline Day 7 & $6.7(1.5-10.9)$ & & \\
\hline
\end{tabular}

*Values are expressed as median (interquartile range). HFpEF: heart failure with preserved ejection fraction; HFrEF: heart failure with reduced ejection fraction.

Table 5. Cost Data

\begin{tabular}{ll}
\hline Clinic visit* & $\$ 1,076(960-1,233)$ \\
Hospital admission* & $\$ 11,471(4,818-26,880)$ \\
Hospital admission per day* & $\$ 1,912(535-2,987)$ \\
Estimated cost saving (per admission prevented) & $\$ 10,395$ \\
\hline
\end{tabular}

*Values are expressed as median (interquartile range).

strategies to provide safe and effective care for our patients and reduce the burden of hospitalizations. Our data support that outpatient IV diuresis, especially when combined with remote tele-monitoring systems, has the potential to significantly improve outpatient care for decompensated HF and reduce the need for HF admissions. This strategy will also be attractive to patients who want to avoid ED visits and hospital admissions due to concerns for high risk exposure to severe acute respiratory syndrome coronavirus 2 (SARS-CoV-2).

\section{Study limitations}

This analysis should be interpreted in the context of several limitations. It was a small single-center retrospective observational study without a comparator arm. Although our results suggest that outpatient IV diuresis is safe and cost-effective, the true efficacy in terms of reducing hospitalizations cannot be evaluated without a control group. This study was done at a tertiary HF center which limits the generalizability to other non-hospital based settings. The majority of patients were male which further limits the generalizability of our findings to the general population. Despite these limitations, these findings are promising and

Table 6. Safety Outcomes

\begin{tabular}{ll}
\hline Arrhythmia & 0 \\
AKI & 0 \\
Hypotension & 0 \\
Severe hypokalemia & $1(3)$ \\
Mild-moderate hypokalemia & $22(65)$ \\
Same-day admission & 1 \\
\hline
\end{tabular}

Values are represented as $\mathrm{n}(\%)$. AKI is defined as increase in Cr by 0.3 $\mathrm{mg} / \mathrm{dL}$. Severe hypokalemia is defined as potassium $<3.0 \mathrm{mmol} / \mathrm{L}$ and mild-moderate hypokalemia as $3.0-3.5 \mathrm{mmol} / \mathrm{L}$. AKI: acute kidney injury. advocate for further investment in innovative ambulatory strategies to manage and prevent decompensated HF.

\section{Conclusions}

In select patients, outpatient IV diuresis is safe and effective, resulting in cost-savings. When combined with tele-monitoring, there is great potential for further reduction of hospitalizations and cost.

\section{Acknowledgments}

None to declare.

\section{Financial Disclosure}

None to declare.

\section{Conflict of Interest}

None to declare.

\section{Informed Consent}

Informed consent was obtained.

\section{Author Contributions}

All authors contributed to concept and design of this study. Vivek Verma and Gavin Hickey analyzed the data. Vivek Verma drafted 
the manuscript. All authors contributed to critically revising the manuscript. All authors gave final approval and agree to be accountable for all aspects of work ensuring integrity and accuracy.

\section{Data Availability}

The authors declare that data supporting the findings of this study are available within the article.

\section{Abbreviations}

ADHF: acute decompensated heart failure; AKI: acute kidney injury; AHF: advanced heart failure; ACE-I: angiotensinconverting enzyme inhibitor; ARB: angiotensin II receptor blocker; Afib: atrial fibrillation; BMP: basic metabolic panel; BB: beta-blocker; BUN: blood urea nitrogen; BNP: brain natriuretic peptide; CRT-D: cardiac resynchronization therapy defibrillator; $\mathrm{CN}$ : charge nurse; $\mathrm{CKD}$ : chronic kidney disease; COPD: chronic obstructive lung disease; CAD: coronary artery disease; $\mathrm{Cr}$ : creatinine; DM2: diabetes mellitus type 2; HF: heart failure; HFpEF: heart failure with preserved ejection fraction; HFrEF: heart failure with reduced ejection fraction; HRRP: Hospital Readmission Reduction Program; HLD: hyperlipidemia; HTN: hypertension; ICD: implantable cardioverter defibrillator; IQR: interquartile range; IV: intravenous; MRA: mineralocorticoid receptor antagonist; NYHA: New York Heart Association; PA: pulmonary artery; SBP: systolic blood pressure

\section{References}

1. Mozaffarian D, Benjamin EJ, Go AS, Arnett DK, Blaha MJ, Cushman M, de Ferranti S, et al. Heart disease and stroke statistics - 2015 update: a report from the American Heart Association. Circulation. 2015;131(4):e29-322.

2. Heidenreich PA, Albert NM, Allen LA, Bluemke DA, Butler J, Fonarow GC, Ikonomidis JS, et al. Forecasting the impact of heart failure in the United States: a policy statement from the American Heart Association. Circ Heart Fail. 2013;6(3):606-619.

3. Dunlay SM, Redfield MM, Weston SA, Therneau TM, Hall Long K, Shah ND, Roger VL. Hospitalizations after heart failure diagnosis a community perspective. J Am Coll Cardiol. 2009;54(18):1695-1702.

4. Gheorghiade M, Zannad F, Sopko G, Klein L, Pina IL, Konstam MA, Massie BM, et al. Acute heart failure syndromes: current state and framework for future research. Circulation. 2005;112(25):3958-3968.

5. Blecker S, Paul M, Taksler G, Ogedegbe G, Katz S. Heart failure-associated hospitalizations in the United States. J Am Coll Cardiol. 2013;61(12):1259-1267.

6. McHugh MD, Ma C. Hospital nursing and 30-day readmissions among Medicare patients with heart failure, acute myocardial infarction, and pneumonia. Med Care. 2013;51(1):52-59.
7. Dunlay SM, Shah ND, Shi Q, Morlan B, VanHouten H, Long KH, Roger VL. Lifetime costs of medical care after heart failure diagnosis. Circ Cardiovasc Qual Outcomes. 2011;4(1):68-75.

8. Okumura N, Jhund PS, Gong J, Lefkowitz MP, Rizkala AR, Rouleau JL, Shi VC, et al. Importance of clinical worsening of heart failure treated in the outpatient setting: evidence from the prospective comparison of ARNI with ACEI to determine impact on global mortality and morbidity in heart failure trial (PARADIGM-HF). Circulation. 2016;133(23):2254-2262.

9. Hospital Readmissions Reduction Program (HRRP) CMS. Accessed July 19, 2020. https://www.cms.gov/ Medicare/Medicare-Fee-for-Service-Payment/AcuteInpatientPPS/Readmissions-Reduction-Program.

10. Buckley LF, Carter DM, Matta L, Cheng JW, Stevens C, Belenkiy RM, Burpee LJ, et al. Intravenous diuretic therapy for the management of heart failure and volume overload in a multidisciplinary outpatient unit. JACC Heart Fail. 2016;4(1):1-8.

11. Hebert K, Dias A, Franco E, Tamariz L, Steen D, Arcement LM. Open access to an outpatient intravenous diuresis program in a systolic heart failure disease management program. Congest Heart Fail. 2011;17(6):309-313.

12. Ryder M, Murphy NF, McCaffrey D, O'Loughlin C, Ledwidge $\mathrm{M}$, McDonald $\mathrm{K}$. Outpatient intravenous diuretic therapy; potential for marked reduction in hospitalisations for acute decompensated heart failure. Eur J Heart Fail. 2008;10(3):267-272.

13. Lazkani M, Ota KS. The role of outpatient intravenous diuretic therapy in a transitional care program for patients with heart failure: a case series. J Clin Med Res. 2012;4(6):434438.

14. Makadia S, Simmons T, Augustine S, Kovell L, Harris C, Chibungu A, Parakh K. The diuresis clinic: a new paradigm for the treatment of mild decompensated heart failure. Am J Med. 2015;128(5):527-531.

15. Durango Luisa F, Katz Stuart D, Li Hung-Kei, Wencker Detlef. Abstract 1891: continuous furosemide infusion is safe and effective in outpatient treatment of refractory heart failure. Circulation. 2006;114:375-376.

16. Ong MK, Romano PS, Edgington S, et al. Effectiveness of remote patient monitoring after discharge of hospitalized patients with heart failure: the better effectiveness after transition - heart failure (BEAT-HF) randomized clinical trial. JAMA Intern Med. 2016;176(3):310-318.

17. Chaudhry SI, Mattera JA, Curtis JP, Spertus JA, Herrin J, Lin Z, Phillips CO, et al. Telemonitoring in patients with heart failure. N Engl J Med. 2010;363(24):2301-2309.

18. Bohm M, Drexler H, Oswald H, Rybak K, Bosch R, Butter C, Klein G, et al. Fluid status telemedicine alerts for heart failure: a randomized controlled trial. Eur Heart J. 2016;37(41):3154-3163.

19. Givertz MM, Stevenson LW, Costanzo MR, Bourge RC, Bauman JG, Ginn G, Abraham WT, et al. Pulmonary artery pressure-guided management of patients with heart failure and reduced ejection fraction. J Am Coll Cardiol. 2017;70(15):1875-1886. 